\title{
Prosthesis-patient mismatch in high-risk patients with severe aortic stenosis: A randomized trial of a self-expanding prosthesis
}

George L. Zorn III, MD, ${ }^{a}$ Stephen H. Little, MD, ${ }^{b}$ Peter Tadros, MD, ${ }^{a}$ G. Michael Deeb, MD, ${ }^{c}$

Thomas G. Gleason, MD, ${ }^{\mathrm{d}}$ John Heiser, MD, ${ }^{\mathrm{e}}$ Neal S. Kleiman, MD, Jae K. Oh, MD, Jeffrey J. Popma, MD, ${ }^{\mathrm{g}}$ David Adams, MD, ${ }^{\mathrm{h}}$ Jian Huang, MD, ${ }^{\mathrm{i}}$ and Michael J. Reardon, MD ${ }^{\mathrm{b}}$

\section{ABSTRACT}

Objectives: We compared the incidence of prosthesis-patient mismatch (PPM) between transcatheter aortic valve replacement (TAVR) using a self-expanding bioprosthesis and surgical aortic valve replacement (SAVR) in the CoreValve US High Risk Pivotal Trial. We sought to determine the influence of PPM on clinical outcomes.

Methods: Patients with severe aortic stenosis and at increased risk for surgery were randomized 1:1 to TAVR or SAVR. Postoperative PPM was defined by the effective orifice area index (EOAi) as severe PPM $\left(\mathrm{EOAi} \leq 0.65 \mathrm{~cm}^{2} / \mathrm{m}^{2}\right)$ and no severe PPM (EOAi $\left.>0.65 \mathrm{~cm}^{2} / \mathrm{m}^{2}\right)$; clinical outcomes were analyzed in the TAVR arm $(n=389)$ and SAVR arm $(n=353)$. Left ventricular mass index and regression were analyzed at baseline and 1 year.

Results: The incidence of severe PPM in the SAVR group at 1 year was $25.7 \%$ versus $6.2 \%$ in the TAVR group $(P<.0001)$. Left ventricular mass index regression at 1 year was $6.8 \%$ for TAVR and $15.1 \%$ for SAVR in patients with severe PPM. At 1 year the rate of all-cause mortality and acute kidney injury were significantly greater in all patients (TAVR + SAVR) with severe PPM compared with no severe PPM $(20.6 \%$ vs $12.0 \%[P=.0145]$ for death and $19.2 \%$ vs $8.5 \%$ $[P=.0008]$ for acute kidney injury).

Conclusions: In patients with high surgical risk and severe aortic stenosis, severe PPM is more common in patients treated with SAVR than those treated with TAVR. Patients with severe PPM are a greater risk for death and acute kidney injury than patients without severe PPM. (J Thorac Cardiovasc Surg 2016;151:1014-23)

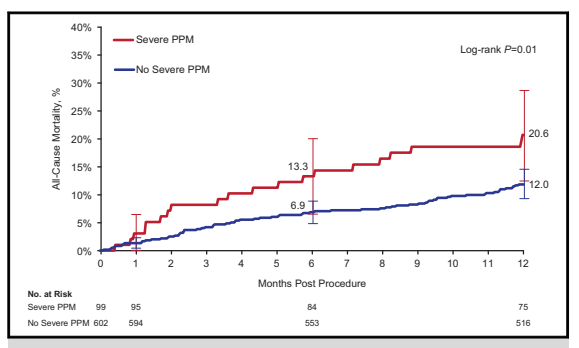

Cumulative incidence of all-cause mortality at 1 year for patients undergoing surgical aortic valve replacement plus transcatheter aortic valve replacement with and without severe prosthesis-patient mismatch (PPM).

\section{Central Message}

Patients with severe PPM are at greater risk for death and acute kidney injury than patients without severe PPM.

\section{Perspective}

Severe prosthesis-patient mismatch (PPM) is significantly more common after surgical aortic valve replacement than after transcatheter aortic valve replacement in patients at high risk with symptomatic severe aortic stenosis. Patients with severe PPM are at a greater risk for death and acute kidney injury than patients without severe PPM.

See Editorial Commentary page 1024.

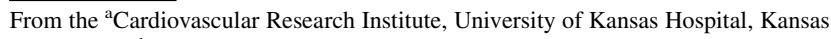
City, Kan; ${ }^{\mathrm{b}}$ Houston Methodist DeBakey Heart and Vascular Center, The Houston Methodist Hospital, Houston, Tex; ${ }^{\mathrm{c}}$ University of Michigan Medical Center, Ann Arbor, Mich; ${ }^{\mathrm{d} U n i v e r s i t y ~ o f ~ P i t t s b u r g h ~ S c h o o l ~ o f ~ M e d i c i n e, ~ P i t t s b u r g, ~ P a ; ~}{ }^{\mathrm{e} S p e c}$ trum Health Hospitals, Grand Rapids, Mich; ${ }^{\mathrm{f}}$ Mayo Clinic, Rochester, Minn; ${ }^{\mathrm{g}}$ Beth Israel Deaconess Medical Center, Boston, Mass; ${ }^{\mathrm{h}}$ Mount Sinai Medical Center, New York, NY; and ${ }^{\mathrm{i}}$ Medtronic, Minneapolis, Minn.

Supported by Medtronic, Minneapolis, Minn.

Read at the 95th Annual Meeting of The American Association for Thoracic Surgery, Seattle, Washington, April 25-29, 2015.

Received for publication April 29, 2015; revisions received Aug 3, 2015; accepted for publication Oct 8, 2015; available ahead of print Nov 21, 2015.

Address for reprints: George L. Zorn III, MD, Cardiovascular Research Institute, University of Kansas Hospital, 3901 Rainbow Blvd, Kansas City, KS 66160 (E-mail: gzorn@kumc.edu).

$0022-5223 / \$ 36.00$

Copyright (c) 2016 by The American Association for Thoracic Surgery

http://dx.doi.org/10.1016/j.jtcvs.2015.10.070
The clinical effects of prosthesis-patient mismatch (PPM) after surgical aortic valve replacement (SAVR) has been debated for the past 3 decades. ${ }^{1,2}$ When a normally functioning prosthetic valve is too small to meet the flow requirements of a specific patient, then a condition of mismatch has occurred. A commonly accepted indicator

Scanning this QR code will take you to supplemental tables for this article. The AATS 2015 Webcast and discussion are available from the URL at the end of the article. 


\section{Abbreviations and Acronyms \\ EOAi $=$ effective orifice area index \\ $\mathrm{LV} \quad=$ left ventricular \\ LVEDD $=$ left ventricular end-diastolic dimension \\ LVM = left ventricular mass \\ LVMI $=$ left ventricular mass index \\ NYHA $=$ New York Heart Association \\ PARTNER $=$ Placement of Aortic Transcatheter Valve Trial \\ PPM = prosthesis-patient mismatch \\ SAVR = surgical aortic valve replacement \\ TAVR $=$ transcatheter aortic valve replacement \\ VARC $=$ Valve Academic Research \\ Consortium-1}

of PPM severity is the effective orifice area calculated by the continuity equation using echocardiography. When divided by the patient's body surface area, an effective orifice area index (EOAi) provides a threshold to define whether severe PPM is present (EOAi $\leq 0.65 \mathrm{~cm}^{2} / \mathrm{m}^{2}$ ) or absent (EOAi $>0.65 \mathrm{~cm}^{2} / \mathrm{m}^{2}$ ). Severe PPM has been associated with higher early ${ }^{3-6}$ and late mortality ${ }^{5,-11}$ after surgical valve replacement; the association of moderate PPM and late clinical outcomes has been less clear. ${ }^{2}$ The effect of PPM on late mortality may be influenced by the presence of obesity, older age, left ventricular (LV) dysfunction, New York Heart Association (NYHA) functional class III or IV symptoms, and concomitant coronary artery bypass grafting. ${ }^{12-14}$

Transcatheter aortic valve replacement (TAVR) has provided another option for reducing PPM in patients undergoing aortic valve replacement due to the lower profile of the transcatheter valve without a sewing ring. ${ }^{15}$ The influence of PPM on late outcomes was examined in 699 patients at high risk for surgery who were randomized to surgery or TAVR in the Placement of Aortic Transcatheter Valve Trial (PARTNER) A trial. ${ }^{14}$ The frequencies of moderate and severe PPM were lower after TAVR than after SAVR in the PARTNER A trial, and these differences were accentuated in those patients with echocardiographic-determined annular diameters $<20 \mathrm{~mm} .{ }^{14}$ This study also identified an important relationship between PPM after surgery and 2-year mortality and left ventricular mass regression. ${ }^{14}$

The design of the CoreValve transcatheter bioprosthesis (Medtronic, Minneapolis, Minn) includes a supraannular porcine pericardial aortic valve that provides durable hemodynamic parameters after implantation. ${ }^{16,17}$ The objectives of this study were to compare the incidence and prognosis of PPM between TAVR using a self-expanding prosthesis and SAVR in the CoreValve US High Risk Pivotal Trial (ClinicalTrials. gov registration identifier: NCT01240902).

\section{METHODS}

\section{Patient Flow and Demographics}

We performed a prospective, randomized multicenter investigation at 45 US clinical sites that enrolled 790 patients who were randomly assigned to treatment with self-expanding TAVR or SAVR. ${ }^{16}$ Inclusion and exclusion criteria have been reported in detail elsewhere; patients at increased surgical risk were considered to have a $>15 \%$ surgical mortality rate at 30 days by 2 clinical site cardiac surgeons. ${ }^{16}$ Severe aortic stenosis was defined as an aortic valve area $\leq 0.8 \mathrm{~cm}^{2}$ or aortic valve index $\leq 0.5 \mathrm{~cm}^{2} / \mathrm{m}^{2}$ and either a mean aortic valve gradient $>40 \mathrm{~mm} \mathrm{Hg}$ or a peak aortic valve velocity $>4.0$ meters per second, at rest or with dobutamine. ${ }^{16}$ Anatomic exclusion criteria included an aortic annular diameter $<18 \mathrm{~mm}$ or $>29 \mathrm{~mm}$.

Patient randomization was stratified by femoral or alternative (subclavian or direct aortic) access for device placement. All patients who were implanted with a CoreValve transcatheter bioprosthesis are included in this analysis. Clinical outcomes and echocardiographic results were collected for $>1$ year. All clinical events were adjudicated by an independent clinical events committee using standardized Valvular Academic Research Consortium-1 (VARC-1) criteria. ${ }^{18}$

\section{Clinical and Echocardiographic Outcomes}

Criteria for major adverse cardiovascular and cerebrovascular events comprising all-cause death; myocardial infarction; all stroke; and reintervention to alter, adjust, or replace a previously implanted valve were adjudicated by an independent clinical events committee using VARC-1 criteria. ${ }^{18}$ Symptom status was assessed using the NYHA functional classification system.

Echocardiograms were evaluated by an independent echocardiographic core lab (Mayo Clinic, Rochester, Minn). Echocardiography measurement methods by the echocardiography core lab have been published. ${ }^{19}$ Specifically, LV stroke volume was calculated by both Doppler (LV outflow track area $\times$ velocity time integral) and 2-dimensional volumetric methods whenever feasible. LV outflow track area was calculated from its diameter at baseline and outer to outer measurement of the bioprosthesis after its implantation. Valvular regurgitation was determined using VARC-1 criteria. $^{18}$

$\mathrm{LV}$ mass $(\mathrm{LVM})$ is determined by the formula $\mathrm{LVM}=0.83 \times[[(\mathrm{LVEDD}$ + LVPW + IVS $\left.\left.)^{3}\right]-\left[(\text { LVEDD })^{3}\right]\right]+0.6$, where LVEDD $=$ LV end-diastolic dimension, LVPW $=\mathrm{LV}$ posterior wall thickness, and IVS $=$ interventricular septal thickness.

\section{Definition of PPM}

PPM was defined as moderate when the EOAi was $\leq 0.85 \mathrm{~cm}^{2} / \mathrm{m}^{2}$ but $>0.65 \mathrm{~cm}^{2} / \mathrm{m}^{2}$ and severe when the EOAi was $\leq 0.65 \mathrm{~cm}^{2} / \mathrm{m}^{2}$. All patients were assessed for either the presence or absence of severe PPM. The first available EOAi at discharge or after was used to define PPM for this analysis.

\section{Statistical Analysis}

Categorical variables were compared with the use of the $\chi^{2}$ or Fisher exact test. Continuous variables were presented as mean \pm standard deviation and compared with the use of the Student $t$ test for independent samples and a paired $t$ test for matched samples. Kaplan-Meier estimates were used to construct the survival curves based on all available followup for the time-to-event analysis. The log-rank test was used to compare the time to events. All testing used a 2-sided alpha level of 0.05 . For selected safety outcomes, the Cox proportional hazard model was used 
TABLE 1. Prosthesis-patient mismatch*

\begin{tabular}{lccc}
\hline EOAi $+\left(\mathbf{c m}^{\left.\mathbf{2} / \mathbf{m}^{2}\right)}\right.$ & $\begin{array}{c}\text { TAVR } \\
(\mathbf{n}=\mathbf{3 8 9})\end{array}$ & $\begin{array}{c}\text { SAVR } \\
(\mathbf{n}=\mathbf{3 5 3})\end{array}$ & $\boldsymbol{P}$ value \\
\hline 1 mo & $\mathrm{n}=344$ & $\mathrm{n}=280$ & \\
Severe PPM & $24(7.0)$ & $58(20.7)$ & $<.0001$ \\
Not severe PPM & $320(93.0)$ & $222(79.3)$ & \\
$\quad$ Moderate PPM & $66(19.2)$ & $88(31.4)$ & \\
$\quad$ None to mild PPM & $254(73.8)$ & $134(47.9)$ & \\
12 mo & $\mathrm{n}=274$ & $\mathrm{n}=206$ & \\
Severe PPM & $17(6.2)$ & $53(25.7)$ & $<.0001$ \\
Not severe PPM & $257(93.8)$ & $153(74.3)$ & \\
$\quad$ Moderate PPM & $57(20.8)$ & $63(30.6)$ & \\
$\quad$ None to mild & $200(73.0)$ & $90(43.7)$ & \\
\hline
\end{tabular}

Values are presented as n (\%).EOAi, Effective orifice area index; TAVR, transcatheter aortic valve replacement; $S A V R$, surgical aortic valve replacement; $P P M$, prosthesispatient mismatch. *Prosthesis-patient mismatch was defined by the first available EOAi at discharge or 1 month. $†$ Severe PPM defined as EOAi $\leq 0.65$, not severe PPM defined as EOAi $>0.65$, moderate PPM defined as $0.65<$ EOAi $\leq 0.85$, and none to mild PPM defined as PPM EOAi $>0.85$.

to compare the difference between severe PPM and no severe PPM in the combined cohort, where treatment (SAVR and TAVR) was included as a covariate. All statistical analyses were performed with the use of SAS software, version 9.2 (SAS Institute, Cary, NC).

\section{RESULTS}

\section{Frequency of PPM and Patient Characteristics}

The incidence of severe PPM in the SAVR group at 1 month was $20.7 \%$ versus $7.0 \%$ in the TAVR group and at 1 year was $25.7 \%$ versus $6.2 \%(P<.0001$ for both time points) (Table 1). At 1 year, moderate PPM was present in $20.8 \%$ of TAVR and $30.6 \%$ of SAVR patients, and no/ mild PPM was present in $73.0 \%$ of TAVR patients and $43.7 \%$ of SAVR patients.

Patients with severe PPM at 1 month had a significantly higher body mass index in both the TAVR and SAVR groups. The TAVR patients with severe PPM had more severe chronic lung disease and home oxygen use than those without severe PPM (Table E1).

\section{Echocardiographic Findings}

Baseline echocardiographic findings are found in Table E2. The EOAi was significantly lower in the severe PPM compared with the no severe PPM patients from both the TAVR $\left(0.32 \pm 0.07\right.$ vs $\left.0.40 \pm 0.12 \mathrm{~cm}^{2} / \mathrm{m}^{2} ; P=.0005\right)$ and the $\operatorname{SAVR}\left(0.36 \pm 0.14\right.$ vs $0.41 \pm 0.12 \mathrm{~cm}^{2} / \mathrm{m}^{2}$; $P=.0109)$ groups. The aortic annulus diameter was significantly less in the severe PPM patients treated by TAVR $(2.07 \pm 0.16$ vs $2.23 \pm 0.21 \mathrm{~cm} ; P=.0006)$ but was only marginally less in the SAVR group with severe PPM (2.14 \pm 0.22 vs $2.19 \pm 0.21 ; P=.0676)$.

At baseline, LVM was significantly greater in the patients with severe PPM than in the patients with no severe PPM treated with SAVR (243.18 vs $224.68 \mathrm{~g} ; P=.0434)$. This difference was not observed in the TAVR patients (severe PPM 226.45 vs no severe PPM $225.95 \mathrm{~g} ; P=.9781$ ).
The deceleration time of the early diastolic mitral valve Doppler inflow (ie, the E wave) was significantly shorter in SAVR patients with PPM than in those with no severe PPM (204.86 vs $236.27 \mathrm{~ms} ; P=.0020$ ) but was not different in TAVR patients with or without severe PPM (231.40 vs $226.60 \mathrm{~ms} ; P=.7812$ ).

Changes in echocardiographic parameters during the 12 months after valve replacement for paired patients are found in Table 2. Both TAVR and SAVR were successful in significantly reducing mean gradient and peak velocity; however, patients with severe PPM in both groups had significantly higher mean gradients (11.18 vs $8.92 \mathrm{~mm} \mathrm{Hg}$ $[P=.0060]$ for TAVR and 16.41 vs $11.32 \mathrm{~mm} \mathrm{Hg}$ $[P<.0001]$ for SAVR) and peak velocities $(2.25$ vs $2.01 \mathrm{~m} / \mathrm{s}[P=.0082]$ for TAVR and 2.68 vs $2.22 \mathrm{~m} / \mathrm{s}$ $[P<.0001]$ for SAVR) compared with the nonsevere PPM patients at 1 year.

LVEDD as measured at 1 year showed a significant difference in the SAVR group between severe PPM and no severe PPM patients (4.97 vs $4.75 \mathrm{~cm} ; P=.0320$ ). This difference was not observed in the TAVR group (4.89 vs $4.98 \mathrm{~cm} ; P=.6516$ ). All SAVR patients had a significant change from baseline in LVEDD at 1 year $(-0.28 \mathrm{~cm} ; P=.0091$ in severe PPM and $-0.27 \mathrm{~cm}$; $P<.0001$ in no severe PPM SAVR patients) that was not observed in the TAVR patients.

The EOAi was significantly greater at 12 months for all TAVR and SAVR patients, but the improvement was significantly greater in the no PPM group compared with the severe PPM group for TAVR $(0.67 \pm 0.29$ vs $0.44 \pm 0.24 ; P=.0064)$ and for SAVR $(0.47 \pm 0.26$ vs $0.31 \pm 0.29 ; P=.0006)$.

At 1 year LVM index was significantly reduced in severe PPM patients in the SAVR $(-22.67 \pm 38.47 \mathrm{~g} ; P=.0019)$ but not the TAVR group $(-11.88 \pm 34.26 \mathrm{~g} ; P=.3288)$. Both the TAVR and the SAVR no severe PPM patients had significant reductions in LV mass index $(P<.0001$ for both) at 1 year. In the patients with severe PPM, LVM index regression at 1 year was $6.8 \%$ for TAVR and $15.1 \%$ for SAVR (Table 2 and Figure 1).

Total aortic regurgitation at 1 month and 1 year is shown in Table E3. Moderate or greater aortic regurgitation was not found in any severe PPM patients at 1 year and was not significantly different between the severe PPM and no severe PPM groups for TAVR or SAVR (Table 2). The presence or absence of aortic regurgitation did not appear to influence the magnitude of LVM regression experienced by patients with severe PPM after SAVR or TAVR (Table E4).

\section{Clinical Outcomes}

Clinical outcomes at 1 year for the SAVR, TAVR, and combined SAVR + TAVR groups in patients with and without severe PPM are found in Table 3. In the combined group the patients with severe PPM had a higher rate of 
TABLE 2. Change in echocardiographic findings from baseline to 12 month follow-up on paired patients

\begin{tabular}{|c|c|c|c|c|c|c|}
\hline \multirow[b]{2}{*}{ Characteristic } & \multicolumn{3}{|c|}{ TAVR } & \multicolumn{3}{|c|}{ SAVR } \\
\hline & $\begin{array}{c}\text { Severe PPM } \\
(\mathbf{n}=\mathbf{2 4})\end{array}$ & $\begin{array}{l}\text { No severe PPM } \\
\quad(\mathbf{n}=\mathbf{3 4 3})\end{array}$ & $P$ value & $\begin{array}{c}\text { Severe PPM } \\
(\mathbf{n}=\mathbf{7 5})\end{array}$ & $\begin{array}{c}\text { No severe PPM } \\
\quad(\mathbf{n}=\mathbf{2 5 9})\end{array}$ & $P$ value \\
\hline Aortic valve peak velocity $(\mathrm{m} / \mathrm{s})$ & $\mathrm{n}=19$ & $\mathrm{n}=270$ & & $\mathrm{n}=47$ & $\mathrm{n}=174$ & \\
\hline Baseline & $4.64(0.63)$ & $4.38(0.56)$ & .0532 & $4.47(0.55)$ & $4.34(0.58)$ & .1787 \\
\hline $1 \mathrm{y}$ & $2.25(0.38)$ & $2.01(0.38)$ & .0082 & $2.68(0.60)$ & $2.22(0.55)$ & $<.0001$ \\
\hline Change & $-2.39(0.49)$ & $-2.37(0.64)$ & .8696 & $-1.79(0.76)$ & $-2.12(0.73)$ & .0060 \\
\hline$P$ value for change from baseline to $1 \mathrm{y}$ & $<.0001$ & $<.0001$ & & $<.0001$ & $<.0001$ & \\
\hline Mean gradient $(\mathrm{mm} \mathrm{Hg})$ & $\mathrm{n}=19$ & $\mathrm{n}=270$ & & $\mathrm{n}=47$ & $\mathrm{n}=174$ & \\
\hline Baseline & $52.63(15.84)$ & $47.74(13.50)$ & .1332 & $50.13(16.41)$ & $47.19(13.69)$ & .2119 \\
\hline $1 \mathrm{y}$ & $11.18(3.92)$ & $8.92(3.41)$ & .0060 & $16.41(7.74)$ & $11.32(6.93)$ & $<.0001$ \\
\hline Change & $-41.45(14.12)$ & $-38.83(13.62)$ & .4193 & $-33.72(18.10)$ & $-35.87(14.68)$ & .3994 \\
\hline$P$ value for change from baseline to $1 \mathrm{y}$ & $<.0001$ & $<.0001$ & & $<.0001$ & $<.0001$ & \\
\hline Aortic valve EOA or valve area $\left(\mathrm{cm}^{2}\right)$ & $\mathrm{n}=13$ & $\mathrm{n}=240$ & & $\mathrm{n}=30$ & $\mathrm{n}=142$ & \\
\hline Baseline & $0.61(0.14)$ & $0.72(0.22)$ & .0757 & $0.75(0.33)$ & $0.76(0.21)$ & .8439 \\
\hline $1 \mathrm{y}$ & $1.45(0.47)$ & $1.94(0.50)$ & .0007 & $1.36(0.54)$ & $1.63(0.46)$ & .0020 \\
\hline Change & $0.84(0.45)$ & $1.22(0.53)$ & .0126 & $0.61(0.59)$ & $0.87(0.46)$ & .0136 \\
\hline$P$ value for change from baseline to $1 \mathrm{y}$ & $<.0001$ & $<.0001$ & & $<.0001$ & $<.0001$ & \\
\hline EOAi $\left(\mathrm{cm}^{2} / \mathrm{m}^{2}\right)$ & $\mathrm{n}=13$ & $\mathrm{n}=240$ & & $\mathrm{n}=40$ & $\mathrm{n}=142$ & \\
\hline Baseline & $0.32(0.08)$ & $0.39(0.11)$ & .0242 & $0.38(0.16)$ & $0.42(0.12)$ & .1684 \\
\hline $1 \mathrm{y}$ & $0.76(0.26)$ & $1.06(0.28)$ & .0002 & $0.69(0.25)$ & $0.89(0.27)$ & $<.0001$ \\
\hline Change & $0.44(0.24)$ & $0.67(0.29)$ & .0064 & $0.31(0.29)$ & $0.47(0.26)$ & .0006 \\
\hline$P$ value for change from baseline to $1 \mathrm{y}$ & $<.0001$ & $<.0001$ & & $<.0001$ & $<.0001$ & \\
\hline $\operatorname{LVEDD}(\mathrm{cm})$ & $\mathrm{n}=12$ & $\mathrm{n}=226$ & & $\mathrm{n}=37$ & $\mathrm{n}=137$ & \\
\hline Baseline & $5.03(0.50)$ & $4.98(0.57)$ & .7697 & $5.25(0.60)$ & $5.02(0.52)$ & .0260 \\
\hline $1 \mathrm{y}$ & $4.89(0.74)$ & $4.98(0.65)$ & .6516 & $4.97(0.63)$ & $4.75(0.53)$ & .0320 \\
\hline Change & $-0.13(0.82)$ & $0.00(0.56)$ & .5806 & $-0.28(0.61)$ & $-0.27(0.64)$ & .9695 \\
\hline$P$ value for change from baseline to $1 \mathrm{y}$ & .5863 & .9248 & & .0091 & $<.0001$ & \\
\hline $\operatorname{LVESD}(\mathrm{cm})$ & $\mathrm{n}=7$ & $\mathrm{n}=184$ & & $\mathrm{n}=29$ & $\mathrm{n}=109$ & \\
\hline Baseline & $3.26(0.54)$ & $3.15(0.76)$ & .7216 & $3.57(0.78)$ & $3.34(0.67)$ & .1049 \\
\hline $1 \mathrm{y}$ & $3.03(0.62)$ & $3.21(0.80)$ & .5440 & $3.28(0.70)$ & $2.99(0.56)$ & .0212 \\
\hline Change & $-0.23(0.72)$ & $0.06(0.71)$ & .2934 & $-0.29(0.65)$ & $-0.34(0.68)$ & .7224 \\
\hline$P$ value for change from baseline to $1 \mathrm{y}$ & .4306 & .2519 & & .0212 & $<.0001$ & \\
\hline $\operatorname{LVM}(\mathrm{g})$ & $\mathrm{n}=9$ & $\mathrm{n}=210$ & & $\mathrm{n}=33$ & $\mathrm{n}=133$ & \\
\hline Baseline & $230.12(41.88)$ & $226.59(65.45)$ & .8730 & $260.33(59.36)$ & $230.93(57.56)$ & .0099 \\
\hline $1 \mathrm{y}$ & $209.35(62.98)$ & $207.64(62.82)$ & .9366 & $216.50(74.23)$ & $188.76(52.35)$ & .0495 \\
\hline Change & $-20.77(66.20)$ & $-18.95(57.86)$ & .9268 & $-43.83(76.01)$ & $-42.17(59.90)$ & .8928 \\
\hline$P$ value for change from baseline to $1 \mathrm{y}$ & .3742 & $<.0001$ & & .0023 & $<.0001$ & \\
\hline LVM index $\left(\mathrm{g} / \mathrm{m}^{2}\right)$ & $\mathrm{n}=9$ & $\mathrm{n}=210$ & & $\mathrm{n}=33$ & $\mathrm{n}=133$ & \\
\hline Baseline & $117.68(20.90)$ & $122.89(32.78)$ & .6370 & $134.47(32.35)$ & $126.00(30.97)$ & .1652 \\
\hline $1 \mathrm{y}$ & $105.80(25.82)$ & $111.95(29.86)$ & .5440 & $111.80(38.58)$ & $102.18(24.53)$ & .1801 \\
\hline Change & $-11.88(34.26)$ & $-10.94(31.13)$ & .9302 & $-22.67(38.47)$ & $-23.82(32.79)$ & .8622 \\
\hline$P$ value for change from baseline to $1 \mathrm{y}$ & .3288 & $<.0001$ & & .0019 & $<.0001$ & \\
\hline LVM index regression at $1 \mathrm{y}(\%)$ & $6.76(31.98)$ & $5.41(27.25)$ & .8849 & $15.08(32.01)$ & $15.72(23.27)$ & .9150 \\
\hline Ejection fraction $(\%)$ & $\mathrm{n}=19$ & $\mathrm{n}=276$ & & $\mathrm{n}=47$ & $\mathrm{n}=175$ & \\
\hline Baseline & $56.84(11.08)$ & $58.72(11.23)$ & .4815 & $54.57(14.02)$ & $57.71(11.80)$ & .1216 \\
\hline $1 \mathrm{y}$ & $61.05(8.09)$ & $59.37(9.90)$ & .4687 & $58.51(8.90)$ & $59.92(8.48)$ & .3178 \\
\hline Change & $4.21(10.04)$ & $0.65(9.92)$ & .1313 & $3.94(11.56)$ & $2.21(10.04)$ & .3113 \\
\hline$P$ value for change from baseline to $1 \mathrm{y}$ & .0841 & .2782 & & .0240 & .0041 & \\
\hline Doppler stroke volume $(\mathrm{mL})$ & $\mathrm{n}=13$ & $\mathrm{n}=244$ & & $\mathrm{n}=40$ & $\mathrm{n}=142$ & \\
\hline Baseline & $69.71(20.47)$ & $76.69(21.49)$ & .2538 & $73.51(16.65)$ & $79.98(21.62)$ & .0820 \\
\hline $1 \mathrm{y}$ & $68.03(23.27)$ & $80.15(22.38)$ & .0588 & $76.76(25.15)$ & $74.98(20.03)$ & .6404 \\
\hline Change & $-1.68(23.34)$ & $3.46(26.13)$ & .4884 & $3.25(26.50)$ & $-4.99(22.60)$ & .0516 \\
\hline$P$ value for change from baseline to $1 \mathrm{y}$ & .7997 & .0399 & & .4426 & .0094 & \\
\hline$\geq$ Moderate total AR $(\%)$ & $\mathrm{n}=19$ & $\mathrm{n}=273$ & & $\mathrm{n}=46$ & $\mathrm{n}=171$ & \\
\hline Baseline & $1(5.3)$ & $15(5.5)$ & $>.9999$ & $2(4.3)$ & $12(7.0)$ & .7391 \\
\hline
\end{tabular}


TABLE 2. Continued

\begin{tabular}{|c|c|c|c|c|c|c|}
\hline \multirow[b]{2}{*}{ Characteristic } & \multicolumn{3}{|c|}{ TAVR } & \multicolumn{3}{|c|}{ SAVR } \\
\hline & $\begin{array}{l}\text { Severe PPM } \\
\quad(\mathbf{n}=\mathbf{2 4})\end{array}$ & $\begin{array}{l}\text { No severe PPM } \\
\quad(\mathbf{n}=\mathbf{3 4 3})\end{array}$ & $P$ value & $\begin{array}{c}\text { Severe PPM } \\
\quad(\mathbf{n}=75)\end{array}$ & $\begin{array}{l}\text { No severe PPM } \\
\quad(\mathbf{n}=\mathbf{2 5 9})\end{array}$ & $P$ value \\
\hline $1 \mathrm{y}$ & $0(0.0)$ & $20(7.3)$ & .2216 & $0(0.0)$ & $3(1.8)$ & $>.9999$ \\
\hline Change no to yes & $0(0.0)$ & $15(5.5)$ & .2942 & $0(0.0)$ & $3(1.8)$ & $>.9999$ \\
\hline Change yes to no & $1(5.3)$ & $10(3.7)$ & .5293 & $2(4.3)$ & $12(7.0)$ & .7391 \\
\hline $\mathrm{E} / \mathrm{e}^{\prime}$ ratio & $\mathrm{n}=11$ & $\mathrm{n}=158$ & & $\mathrm{n}=29$ & $\mathrm{n}=106$ & \\
\hline Baseline & $27.50(13.35)$ & $24.79(12.77)$ & .4978 & $25.34(12.26)$ & $23.14(10.13)$ & .3258 \\
\hline $1 \mathrm{y}$ & $25.03(15.82)$ & $26.09(12.75)$ & .7945 & $23.83(9.56)$ & $22.03(9.95)$ & .3839 \\
\hline Change & $-2.46(12.75)$ & $1.30(11.35)$ & .2927 & $-1.50(11.24)$ & $-1.12(10.09)$ & .8585 \\
\hline$P$ value for change from baseline to $1 \mathrm{y}$ & .5362 & .1511 & & .4773 & .2573 & \\
\hline Mitral E/A ratio & 14 & 195 & & 27 & 107 & \\
\hline Baseline & $1.06(0.44)$ & $1.13(0.74)$ & .6357 & $1.34(0.96)$ & $1.01(0.55)$ & .1041 \\
\hline $1 \mathrm{y}$ & $0.87(0.33)$ & $1.03(0.61)$ & .1008 & $1.10(0.45)$ & $0.91(0.33)$ & .0557 \\
\hline Change & $-0.20(0.24)$ & $-0.09(0.85)$ & .2454 & $-0.24(0.86)$ & $-0.10(0.48)$ & .4241 \\
\hline$P$ value for change from baseline to $1 \mathrm{y}$ & .0090 & .1269 & & .1623 & .0344 & \\
\hline Deceleration time of E (ms) & 13 & 190 & & 36 & 125 & \\
\hline Baseline & $236.08(79.21)$ & $233.91(74.57)$ & .9197 & $197.92(59.40)$ & $234.22(81.80)$ & .0042 \\
\hline $1 \mathrm{y}$ & $249.46(91.02)$ & $255.05(83.96)$ & .8177 & $214.61(76.88)$ & $240.70(76.29)$ & .0730 \\
\hline Change & $13.38(73.60)$ & $21.14(83.50)$ & .7448 & $16.69(68.91)$ & $6.48(73.23)$ & .4561 \\
\hline$P$ value for change from baseline to $1 \mathrm{y}$ & .5244 & .0006 & & .1550 & .3246 & \\
\hline Septal thickness $(\mathrm{mm})$ & $\mathrm{n}=10$ & $\mathrm{n}=222$ & & $\mathrm{n}=33$ & $\mathrm{n}=137$ & \\
\hline Baseline & $12.00(1.70)$ & $11.91(2.20)$ & .9035 & $12.79(2.01)$ & $12.28(1.89)$ & .1707 \\
\hline $1 \mathrm{y}$ & $11.60(2.01)$ & $11.32(2.14)$ & .6846 & $12.09(2.35)$ & $11.39(2.29)$ & .1164 \\
\hline Change & $-0.40(1.90)$ & $-0.59(2.47)$ & .8065 & $-0.70(2.02)$ & $-0.89(2.40)$ & .6690 \\
\hline$P$ value for change from baseline to $1 \mathrm{y}$ & .5217 & .0004 & & .0565 & $<.0001$ & \\
\hline Posterior wall thickness (mm) & $\mathrm{n}=10$ & $\mathrm{n}=217$ & & $\mathrm{n}=37$ & $\mathrm{n}=136$ & \\
\hline Baseline & $11.50(1.35)$ & $11.18(1.91)$ & .6015 & $11.51(1.77)$ & $11.27(1.94)$ & .4833 \\
\hline $1 \mathrm{y}$ & $10.40(1.65)$ & $10.47(1.90)$ & .9027 & $10.57(2.38)$ & $10.23(1.73)$ & .4203 \\
\hline Change & $-1.10(1.73)$ & $-0.71(2.21)$ & .5782 & $-0.95(2.58)$ & $-1.04(2.22)$ & .8305 \\
\hline$P$ value for change from baseline to $1 \mathrm{y}$ & .0751 & $<.0001$ & & .0321 & $<.0001$ & \\
\hline Relative wall thickness & $\mathrm{n}=10$ & $\mathrm{n}=216$ & & $\mathrm{n}=37$ & $\mathrm{n}=135$ & \\
\hline Baseline & $0.46(0.07)$ & $0.45(0.10)$ & .7599 & $0.45(0.09)$ & $0.46(0.09)$ & .6311 \\
\hline $1 \mathrm{y}$ & $0.43(0.09)$ & $0.43(0.10)$ & .9835 & $0.43(0.12)$ & $0.44(0.09)$ & .8801 \\
\hline Change & $-0.03(0.11)$ & $-0.03(0.12)$ & .8171 & $-0.01(0.12)$ & $-0.02(0.12)$ & .8168 \\
\hline$P$ value for change from baseline to $1 \mathrm{y}$ & .3533 & .0018 & & .5505 & .0887 & \\
\hline
\end{tabular}

Categorical variables are presented as n (\%); continuous variables are mean (SD). TAVR, Transcatheter aortic valve replacement; SAVR, surgical aortic valve replacement; $P P M$, prosthesis-patient mismatch; $E O A$, effective orifice area; $E O A i$, effective orifice area index; $L V E D D$, left ventricular end diastolic dimension; $L V E S D$, left diastolic end systolic dimension; $L V M$, left ventricular mass; $A R$, aortic regurgitation; $E / e^{\prime}$, early mitral inflow velocity/mitral annulus early diastolic velocity; $E / A$, earl mitral inflow velocity/late mitral inflow velocity.

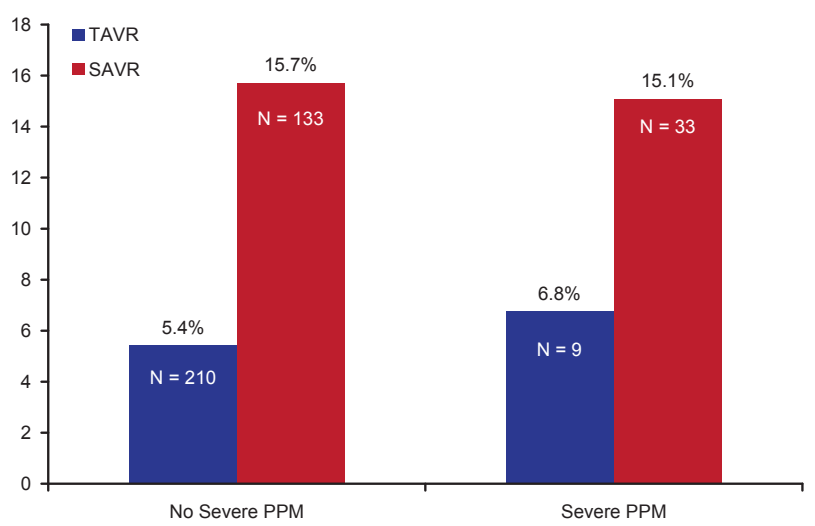

FIGURE 1. Percent left ventricular mass regression at 1 year. TAVR, Transcatheter aortic valve replacement; SAVR, surgical aortic valve replacement; $P P M$, prosthesis-patient mismatch. all-cause mortality (20.6\% vs $12.0 \% ; P=.0145)$ (Figure 2$)$. When adjusted for treatment differences between TAVR and SAVR overall, the $P$ value was .0666 for the difference in all-cause mortality between severe and no severe PPM.

Acute kidney injury was more commonly seen with severe PPM in the SAVR patients $(21.3 \%$ vs $12.4 \%$; $P=.0476)$. In the TAVR group, severe PPM was increasingly involved with acute kidney injury, but this did not achieve statistical significance $(12.5 \%$ vs $5.5 \% ; P=.1633)$.

NYHA functional class III or IV improved similarly regardless of PPM status in the TAVR and the SAVR groups (Table 4). The severe PPM TAVR group was $91.7 \%$ at baseline and improved to $5.3 \%$ at 1 year, whereas the severe PPM SAVR patients were $81.1 \%$ at baseline and $10.4 \%$ NYHA functional class III or IV at 1 year. 
TABLE 3. Cumulative incidence of clinical outcomes to 1 year

\begin{tabular}{|c|c|c|c|c|c|c|c|c|c|}
\hline \multirow[b]{2}{*}{ Characteristic } & \multicolumn{3}{|c|}{ Overall } & \multicolumn{3}{|c|}{ TAVR } & \multicolumn{3}{|c|}{ SAVR } \\
\hline & $\begin{array}{l}\text { Severe PPM* } \\
\quad(\mathbf{n}=99)\end{array}$ & $\begin{array}{l}\text { No severe PPM* } \\
\quad(\mathbf{n}=\mathbf{6 0 2})\end{array}$ & $\begin{array}{c}P \\
\text { value }\end{array}$ & $\begin{array}{l}\text { Severe PPM* } \\
\quad(\mathbf{n}=\mathbf{2 4})\end{array}$ & $\begin{array}{l}\text { No severe PPM* } \\
\quad(\mathbf{n}=\mathbf{3 4 3})\end{array}$ & $\begin{array}{c}P \\
\text { value }\end{array}$ & $\begin{array}{l}\text { Severe PPM* } \\
\quad(\mathbf{n}=\mathbf{7 5})\end{array}$ & $\begin{array}{c}\text { No severe PPM* } \\
\quad(\mathbf{n}=\mathbf{2 5 9})\end{array}$ & $\begin{array}{c}P \\
\text { value }\end{array}$ \\
\hline All-cause mortality & 20.6 & 12.0 & $.0145 \dagger$ & 16.9 & 10.0 & .2436 & 21.8 & 14.5 & .1358 \\
\hline Cardiovascular mortality & 11.7 & 8.2 & .2326 & 9.1 & 7.4 & .7696 & 12.5 & 9.2 & .3882 \\
\hline All stroke & 8.4 & 10.1 & .6756 & 0.0 & 8.3 & .1623 & 11.1 & 12.6 & .8184 \\
\hline Myocardial infarction & 0.0 & 1.7 & .2068 & 0.0 & 1.8 & .5237 & 0.0 & 1.6 & .2849 \\
\hline Reintervention & 0.0 & 0.9 & .3863 & 0.0 & 1.5 & .5706 & 0.0 & 0.0 & NA \\
\hline $\begin{array}{l}\text { Life-threatening or } \\
\text { disabling bleeding }\end{array}$ & 33.6 & 24.3 & .0521 & 26.5 & 13.5 & .1128 & 35.8 & 38.5 & .6817 \\
\hline $\begin{array}{l}\text { Major vascular } \\
\text { complication }\end{array}$ & 3.0 & 4.2 & .6004 & 4.2 & 6.1 & .6993 & 2.7 & 1.5 & .5184 \\
\hline Valve thrombosis & 0.0 & 0.0 & NA & 0.0 & 0.0 & NA & 0.0 & 0.0 & NA \\
\hline $\begin{array}{l}\text { Aortic valve } \\
\text { hospitalization }\end{array}$ & 17.8 & 14.0 & .3515 & 22.0 & 15.6 & .4169 & 16.3 & 11.7 & .3141 \\
\hline Acute kidney injury & 19.2 & 8.5 & $.0008 \dagger$ & 12.5 & 5.5 & .1633 & 21.3 & 12.4 & .0476 \\
\hline
\end{tabular}

Values are presented as \%. TAVR, Transcatheter aortic valve replacement; SAVR, surgical aortic valve replacement; PPM, prosthesis-patient mismatch; NA, not applicable. *PPM was defined as severe PPM when the external orifice area index was $\leq 0.65 \mathrm{~cm}^{2} / \mathrm{m}^{2}$; no severe PPM was defined as external orifice area index $>0.65 \mathrm{~cm} / \mathrm{m}^{2}$. $\dagger$ Adjusted $P=.0860$ for all-cause mortality or major stroke; adjusted $P=.0666$ for all-cause mortality; and adjusted $P=.0230$ for acute kidney injury.

\section{DISCUSSION}

There are 3 principal findings from this study. First, PPM is more common with SAVR than TAVR in this randomized trial of a self-expanding prosthesis in the treatment of aortic stenosis in high-risk patients. Second, LVM regression is less robust in the treatment group receiving TAVR compared with SAVR. Third, we observed that patients with severe PPM have a higher rate of all-cause mortality and acute kidney injury compared with patients without severe PPM.
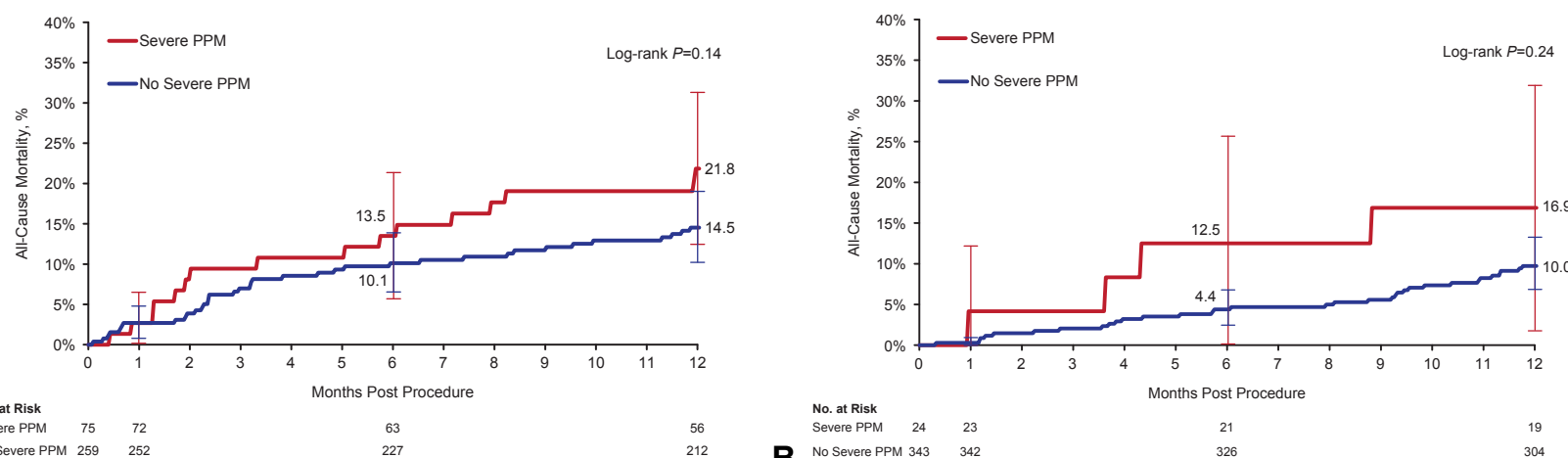

\begin{tabular}{lll} 
Severe PPM & 75 & 72 \\
\hline & & \\
\hline
\end{tabular}

227

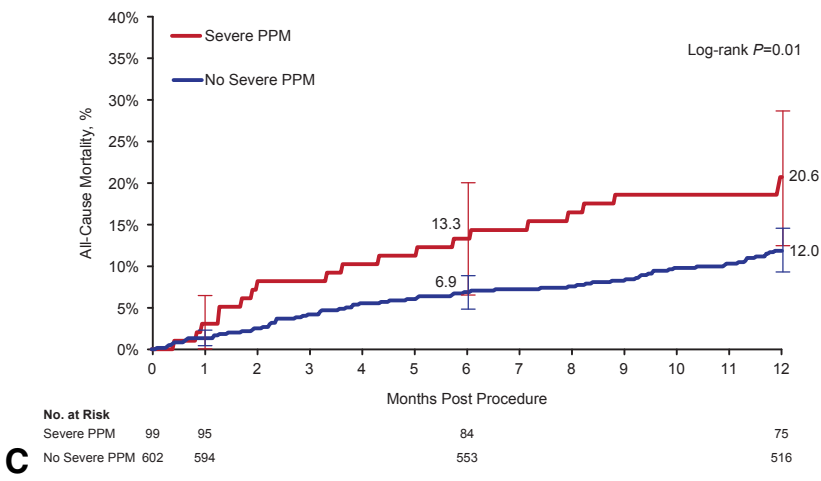

FIGURE 2. Cumulative incidence of all-cause mortality at 1 year for A, surgical aortic valve replacement, B, transcatheter aortic valve replacement, and C, patients undergoing combined transcatheter aortic valve replacement plus surgical aortic valve replacement. PPM, Prosthesis-patient mismatch. 
TABLE 4. New York Heart Association functional classification III or IV over time

\begin{tabular}{|c|c|c|c|c|c|c|}
\hline \multirow[b]{2}{*}{ Time } & \multicolumn{3}{|c|}{ TAVR } & \multicolumn{3}{|c|}{ SAVR } \\
\hline & $\begin{array}{c}\text { Severe PPM } \\
(\mathrm{n}=\mathbf{2 4}) \\
\end{array}$ & $\begin{array}{c}\text { No severe PPM } \\
\quad(n=343)\end{array}$ & $P$ value & $\begin{array}{c}\text { Severe PPM } \\
(\mathbf{n}=\mathbf{7 5}) \\
\end{array}$ & $\begin{array}{c}\text { No severe PPM } \\
\quad(\mathbf{n}=\mathbf{2 5 9}) \\
\end{array}$ & $P$ value \\
\hline Baseline & $91.7(22 / 24)$ & $82.5(283 / 343)$ & .2470 & $81.1(60 / 74)$ & $81.8(207 / 253)$ & .8854 \\
\hline $1 \mathrm{mo}$ & $21.7(5 / 23)$ & $12.7(42 / 331)$ & .2161 & $27.7(18 / 65)$ & $21.7(52 / 240)$ & .3055 \\
\hline $6 \mathrm{mo}$ & $10.5(2 / 19)$ & $6.6(20 / 305)$ & .5046 & $6.9(4 / 58)$ & $5.5(11 / 200)$ & .6890 \\
\hline $1 \mathrm{y}$ & $5.3(1 / 19)$ & $5.3(15 / 282)$ & .9916 & $10.4(5 / 48)$ & $5.5(10 / 183)$ & .2152 \\
\hline
\end{tabular}

Values are presented as $\%(\mathrm{n} / \mathrm{N}) . T A V R$, Transcatheter aortic valve replacement; SAVR, surgical aortic valve replacement; $P P M$, prosthesis-patient mismatch.

\section{Comparative Incidence of PPM}

TAVR has provided another option for reducing PPM in patients undergoing aortic valve replacement due to the lower profile of the transcatheter valve without a sewing ring. ${ }^{15} \mathrm{~A}$ matched analysis of patients with aortic stenosis compared 50 patients treated using a balloon-expandable transcatheter valve with two groups of 50 patients who underwent surgery with a stented valve (Perimount Magna; Edwards Lifesciences, Irvine, Calif) or a stentless valve (Freestyle; Medtronic). ${ }^{15}$ The incidence of severe PPM was significantly lower in the TAVR group $(6 \%)$ than in the stented $(28 \%)$ or stentless $(20 \%)$ surgical groups, albeit with a higher rate of moderate paravalvular regurgitation with TAVR $(8 \%)$ than surgery $(0 \%) .{ }^{15}$ Pibarot and colleagues $^{14}$ evaluated the influence of PPM on late outcomes in 699 patients at "high-risk" for surgery who were randomized to surgery or TAVR in the PARTNER A trial. ${ }^{14}$ Patients in the TAVR group were exclusively treated with the balloon-expandable Sapien (Edwards Lifesciences) bioprosthesis. Similar to ours and other studies, the frequency of severe PPM was lower after TAVR $(19.7 \%)$ than after SAVR $(28.1 \%)$, although the severe PPM rates were higher than our experience in the current randomized CoreValve trial. ${ }^{1}$

\section{LVM Regression}

At baseline both treatment groups were similar with no significant difference in LVEDD, LVESD, LV end diastolic volume, or LV end systolic volume. Patients with severe PPM after SAVR had larger baseline LVM compared with those patients without severe PPM after SAVR and compared with TAVR-treated patients with or without severe PPM.

Comparing patients with severe PPM with those without severe PPM in the TAVR treatment arm, there was no significant difference in LVM index at baseline, LVM index at 1 year, or in the magnitude of LVM index regression at 1 year. Within the SAVR treatment group, the comparison of patients with severe PPM to those without severe PPM demonstrated a similar lack of difference in LVM index at baseline, LVM index at 1 year, or in the magnitude of LVM index regression at 1 year.

Although severe PPM or no severe PPM did not appear to influence LVM index within each treatment group, there were notable differences in LVM index regression between the TAVR and SAVR treatment arms, irrespective of the finding of severe PPM. Within the TAVR group the LVM index regression was $6.8 \%$ with severe PPM and $5.4 \%$ without severe PPM. Within the SAVR group the LVM index regression was $15.1 \%$ with severe PPM and $15.7 \%$ without severe PPM. We hypothesized that the higher incidence of aortic valve regurgitation in the TAVR treatment group may have adversely influenced LVM index regression at 1 year. However, within both the TAVR and SAVR treatment groups the finding of moderate or greater aortic valve regurgitation was not associated with reduced LVM index regression. It is important to note the importance of LVEDD and wall thickness measures in the calculation of LVM. The change in wall thickness was not different between SAVR and TAVR groups, and only LVEDD was relatively smaller after SAVR. Because LVEDD can be profoundly affected by LV preload, which could be reduced on the basis of acute right ventricular dysfunction after SAVR, it is possible that the calculated LVM may not be the most representative measure of myocardial remodeling. This question is the subject of ongoing studies that use cardiac magnetic resonance imaging to provide a more robust measure of LVM before and after aortic stenosis intervention. Although the apparent influence of severe PPM on LV remodeling at 1 year was unremarkable within each treatment group, the echocardiographic diagnosis of severe PPM was nonetheless associated with an increased risk of mortality at 1-year follow-up.

\section{Severe PPM Outcomes}

PPM has been associated with higher early ${ }^{3-6}$ and late mortality $^{5,-11}$ after aortic valve surgery. In a metaanalysis comprising 34 studies that included 27,186 patients and 133,141 patient-years, both moderate and severe PPM increased all-cause mortality (hazard ratio, 1.19 and 1.84, respectively) and cardiac-related mortality (hazard ratio, 1.32 and 6.46, respectively); these relationships were consistent over long-term follow-up out to 12 years. ${ }^{20}$

Our TAVR patients with severe PPM did not demonstrate a significant difference in all-cause mortality at 1 year (16.9\% vs $10.0 \% ; P=.2436)$. Similarly, mortality in SAVR patients with and without severe PPM trended but did not achieve statistical significance $(21.8 \%$ vs $14.5 \%$; 
$P=.1358$ ). Grouping our TAVR and SAVR patients together demonstrated a significant mortality increase in severe PPM compared with no severe PPM $(P=.0145)$. We believe these results are consistent with previous studies concluding that severe PPM matters. It is just a matter of having adequate patient numbers.

\section{Study Limitations}

This study included data from a large, multi-institutional, randomized trial comparing TAVR with contemporary open surgical results. Our study is a post hoc analysis of 12-month data and thus is not powered for these subgroup analyses. The comparison between severe PPM and no severe PPM groups was not adjusted for differences in other patient variables. Although the demographic characteristics of the severe PPM and no severe PPM patients were essentially the same between the groups, there were a couple of differences in baseline echocardiographic parameters (Tables E1 and E2). Subsequent evaluations will benefit from the long-term follow-up of the CoreValve trial.

Although this represents the largest randomized trial of a self-expanding valve in the treatment of aortic stenosis, our analysis remains limited by patient numbers. There were too few patients with severe PPM in the TAVR treatment group to allow meaningful comparisons of some clinical and echocardiographic outcomes.

\section{Clinical Implications}

Patients with severe PPM have increased all-cause mortality and acute kidney injury. TAVR using a selfexpanding valve decreases the incidence of PPM in high-risk patients with severe aortic stenosis. Longer-term studies are necessary to better understand LV mass regression for patients with severe PPM.

\section{Conflict of Interest Statement}

George L. Zorn, III, reports receiving consulting fees from Medtronic and Edwards Lifesciences (Irvine, Calif). Stephen Little has received lecture fees from St Jude Medical (St Paul, Minn) and grant support from Seimens Healthcare (Erlangen, Germany) and St Jude Medical. Peter Tadros has received consulting fees from Medtronic and St Jude Medical. G. Michael Deeb serves as an advisor and consultant to Medtronic. Thomas G. Gleason has received institutional research grant support from Medtronic. Neal S. Kleiman has received grant support from Medtronic. Jae K. Oh reports research support for the echocardiographic core laboratory from Medtronic. Jeffrey J. Popma has received institutional research grants from Boston Scientific (Natick, Mass), Direct Flow Medical (Santa Rosa, Calif), and Medtronic. David Adams has royalty agreements through Mount Sinai School of Medicine with Edwards Lifesciences and Medtronic and has received institutional grant support from Medtronic. Jian Huang is an employee and shareholder of Medtronic. Michael Reardon reports receiving consulting fees from Medtronic. John Heiser has nothing to disclose with regard to commercial support.

You can watch a Webcast of this AATS meeting presentation by going to: http://webcast.aats.org/2015/Video/ Monday/04-27-15_4E_1420_Zorn.mp4.

The authors thank Colleen Gilbert, PharmD, for providing editorial support and Mike Boulware, $\mathrm{PhD}$, for providing critical review of the manuscript. Both are employees of Medtronic.

\section{References}

1. Rahimtoola SH. The problem of valve prosthesis-patient mismatch. Circulation. 1978;58:20-4.

2. Daneshvar SA, Rahimtoola SH. Valve prosthesis-patient mismatch (VP-PM): a long-term perspective. J Am Coll Cardiol. 2012;60:1123-35.

3. Blackstone EH, Cosgrove DM, Jamieson WR, Birkmeyer NJ, Lemmer JH Jr, Miller DC, et al. Prosthesis size and long-term survival after aortic valve replacement. J Thorac Cardiovasc Surg. 2003;126:783-96.

4. Astudillo LM, Santana O, Urbandt PA, Benjo AM, Elkayam LU, Nascimento FO, et al. Clinical predictors of prosthesis-patient mismatch after aortic valve replacement for aortic stenosis. Clinics. 2012;67:55-60.

5. Walther T, Rastan A, Falk V, et al. Patient prosthesis mismatch affects short- and long-term outcomes after aortic valve replacement. Eur J Cardiothorac Surg. 2006;30:15-9

6. Blais C, Dumesnil JG, Baillot R, Simard S, Doyle D, Pibarot P. Impact of valve prosthesis-patient mismatch on short-term mortality after aortic valve replacement. Circulation. 2003;108:983-8.

7. Florath I, Albert A, Rosendahl U, Ennker IC, Ennker J. Impact of valve prosthesis-patient mismatch estimated by echocardiographic-determined effective orifice area on long-term outcome after aortic valve replacement. Am Heart J. 2008;155:1135-42.

8. Kohsaka S, Mohan S, Virani S, Lee VV, Contreras A, Reul GJ, et al. Prosthesispatient mismatch affects long-term survival after mechanical valve replacement. J Thorac Cardiovasc Surg. 2008;135:1076-80.

9. Rao V, Jamieson WR, Ivanov J, Armstrong S, David TE. Prosthesis-patient mismatch affects survival after aortic valve replacement. Circulation. 2000; 102:III5-9.

10. Mohty D, Malouf JF, Girard SE, Schaff HV, Grill DE, Enriquez-Sarano ME, et al Impact of prosthesis-patient mismatch on long-term survival in patients with small St Jude Medical mechanical prostheses in the aortic position. Circulation. 2006;113:420-6.

11. Tasca G, Mhagna Z, Perotti S, Centurini PB, Sabatini T, Amaducci A, et al. Impact of prosthesis-patient mismatch on cardiac events and midterm mortality after aortic valve replacement in patients with pure aortic stenosis. Circulation 2006;113:570-6.

12. Tully PJ, Aty W, Rice GD, Bennetts JS, Knight JL, Baker RA. Aortic valve prosthesis-patient mismatch and long-term outcomes: 19-year single-center experience. Ann Thorac Surg. 2013;96:844-50.

13. Ruel M, Al-Faleh H, Kulik A, Chan KL, Mesana TG, Burwash IG. Prosthesispatient mismatch after aortic valve replacement predominantly affects patients with preexisting left ventricular dysfunction: effect on survival, freedom from heart failure, and left ventricular mass regression. J Thorac Cardiovasc Surg. 2006;131:1036-44

14. Pibarot P, Weissman NJ, Stewart WJ, Hahn RT, Lindman BR, McAndrew T, et al Incidence and sequelae of prosthesis-patient mismatch in transcatheter versus surgical valve replacement in high-risk patients with severe aortic stenosis: a PARTNER trial cohort-A analysis. J Am Coll Cardiol. 2014;64 1323-34.

15. Clavel MA, Dumont E, Pibarot P, Doyle D, De Larochellière R, Villeneuve J, et al. Severe valvular regurgitation and late prosthesis embolization after percutaneous aortic valve implantation. Ann Thorac Surg. 2009;87:618-21.

16. Adams DH, Popma JJ, Reardon MJ. Transcatheter aortic-valve replacement with a self-expanding prosthesis. N Engl J Med. 2014;371:967-8.

17. Popma JJ, Adams DH, Reardon MJ, Yakubov SJ, Kleiman NS, Heimansohn D, et al. Transcatheter aortic valve replacement using a self-expanding bioprosthesis 
in patients with severe aortic stenosis at extreme risk for surgery. J Am Coll Cardiol. 2014;63:1972-81.

18. Leon MB, Piazza N, Nikolsky E, Blackstone EH, Cutlip DE, Kappetein AP, et al. Standardized endpoint definitions for transcatheter aortic valve implantation clinical trials: a consensus report from the Valve Academic Research Consortium. J Am Coll Cardiol. 2011;57:253-69.

19. Oh JK, Little SH, Abdelmoneim SS, Reardon MJ, Kleiman NS, Lin G, et al. Regression of paravalvular aortic regurgitation and remodeling of selfexpanding transcatheter aortic valve: an observation from the CoreValve US Pivotal Trial. JACC Cardiovasc Imag. October 16, 2015 [Epub ahead of print]. 20. Head SJ, Mokhles MM, Osnabrugge RL, Pibarot P, Mack MJ, Takkenberg JJ, et al. The impact of prosthesis-patient mismatch on long-term survival after aortic valve replacement: a systematic review and meta-analysis of 34 observational studies comprising 27186 patients with 133141 patient-years. Eur Heart J. 2012;33:1518-29.

Key Words: aortic stenosis, transcatheter aortic valve replacement, prosthesis-patient mismatch

\section{Discussion}

Dr Craig Smith (New York, NY). I have 1 disclosure. As the surgical principal investigator of the Placement of Aortic Transcatheter Valve (PARTNER) trial, I receive reimbursement from the sponsor for travel and other expenses related to trial management.

That was a very nice subset analysis on patientprosthesis mismatch (PPM). If nothing else, this adds to the accumulating evidence that transcatheter aortic valve replacement (TAVR) offers advantages in orifice area in small roots regardless of risk profile. But I have a cautionary observation and 3 questions.

One of the 3 major conclusions is that PPM is about fourfold more common following surgical aortic valve replacement than TAVR at 1 year: 25.7 versus 6.2. It is not surprising that the authors could not resist a direct comparison to results from the PARTNER trial, which I appreciate you sharing in advance. Although the surgical arms in the 2 trials had very similar frequency of severe PPM, about $26 \%$ versus 28\%, severe PPM was lower in the CoreValve TAVR group than PARTNER, at $6.2 \%$ versus $19.7 \%$.

I can certainly understand the desire to emphasize this seeming competitive advantage of 1 platform over another, but it is important to remember that PARTNER treated higher-risk patients with average risk scores and 1-year all-cause mortality that was about $50 \%$ higher than the somewhat lower-risk patients in the CoreValve trial. So they are just different populations.

They were also not contemporaneous. The nonrandomized continuous access cohort of the PARTNER trial was somewhat more contemporaneous, and also a little bit lower risk; the Society of Thoracic Surgeons score was a little closer to 10 . In that cohort, the severe PPM incidence fell to about $13.6 \%$.

Even more importantly, the 2 trials used different core labs, which leads to my first question: At what level was the orifice measured? Specifically, was it measured at the inflow end of the valve, or right below the visible valve hinges, closer to the annulus?

Dr Zorn. I can't answer that question directly about the PARTNER trial.

Dr Smith. There is debate about this, and I gather the 2 core labs differ on this point. If it is measured at the inflow level, the orifice will be larger than if it is measured right below the leaflets, and I believe the 2 trials differ on this point, so it is a potential source of ostensibly larger orifice areas in the CoreValve trial. That is something we can let the echocardiographers settle.

Dr Zorn. Let me comment on that briefly. They were both core lab-reviewed trials and I agree with you. I do not want to make any strong comparisons across trials here.

Dr Smith. I understand.

Dr Zorn. We were concerned about the issue of measuring and inconsistencies in a calculated number.

Dr Smith. Understood. The authors also show that PPM correlated with late mortality. Totally unsurprising; it is what would be expected. And it was only significant when the surgical and TAVR arms were combined.

Another unique feature of the CoreValve trial that you touched on is that the surgical aortic valve replacement (SAVR) mortality at 1 year was significantly higher than the TAVR mortality. Is it possible that whatever accounts for that mortality imbalance is also distorting the PPM correlation with mortality? Acknowledging that sample size might be a big problem here, was a Cox regression analysis considered or done to test for interaction between PPM and procedure type?

Dr Zorn. It was. In fact, we did a Cox regression analysis accounting for the different therapies that had a $P$ value of .086 .

Dr Smith. So close, but not.

Dr Zorn. Correct, when comparing for those.

Dr Smith. Okay. Thank you. Finally, Table 1 shows that there were 24 patients in the TAVR group who had severe PPM at 1 month that decreased to 17 at 12 months, which is a departure of 7 patients, or about $29 \%$ of the group. I hope I am reading that correctly. Were these mortalities? It does not appear that they were downgraded, because the moderate group does not change, and it would be surprising if it did. In contrast, the severe PPM SAVR group decreased by a relatively modest 5 patients, from 58 to 53 , which is about $9 \%$.

Is the higher proportional loss from the TAVR group in the severe PPM category accentuating the differences between the groups? And if the departures were all mortalities, as I assume they were, what accounts for what appears to be a differential influence of PPM in the TAVR group?

Dr Zorn. They were not all mortalities. The difference in numbers - and I would have to look specifically at the chart 
that we are talking about in the manuscript - a number of those, if we did not have paired analysis at both 1 month and 1 year, those patients were not used in certain analyses. So if patients were not available for both studies, we did not use their data for the paired analysis but we did for some of the mean variables.

Dr Smith. That probably was there and I missed it, but those patients are still subtractions from the total.

Dr Michael Reardon (Houston, Tex). Also, we did not measure effective orifice area because it is a calculated value. We measured at the inflow for the left ventricular outflow tract.

Two things, Craig. First of all, we should not compare these trials; they are not meant to be compared at all. The CoreValve trial was just to look at CoreValve versus surgery, not to compare CoreValve to PARTNER. They are 2 separate and completely different things.

When you look at these, you are better off looking at mean gradients because we actually measure those, whereas effective orifice area is a calculated value, much like left ventricular mass is a calculated value that is greatly influenced by a cube of left ventricular end diastolic dimension.

Dr Niv Ad (Falls Church, Va). Excellent presentation. I wonder if in lieu of the inability to show the differences with PPM you really made the right analysis. In other words, the role of diastolic dysfunction, especially in patients with residual aortic insufficiency, should be emphasized more than it is, because this might put some light on why patients in the TAVR group are not doing as well as we expected when they do not have PPM.

To be able to fully answer the question you need to look at diastolic dysfunction as well.

Dr Zorn. I agree with you. To that end, Steve Little is working on a manuscript that I believe has been submitted where he spends a lot of time looking at that exact issue. It was not the focus of our study, but Little's work is going to look a lot at diastolic dysfunction and its results.

Dr Pasquale Totaro (Palermo, Italy). Congratulations for your presentation. I am a little bit surprised by the relatively high incidence of severe and moderate PPM in the surgical group. Can you tell us something more about the choice of the prosthesis-if anything was done intraoperatively to prevent this? And also just another comment: You showed us a significant reduction in left ventricular mass regression in the surgical group, but especially in patients with severe PPM. Do you have any idea or comments about that?

Dr Zorn. Let me answer the first question. There was no restriction on use of surgical valves in the trial. So surgeons were free to choose their valves. The majority of valves used were 21 and 23; there was a relatively small use of
25 and 27 valves for the trial. They were encouraged not to enroll patients who would require annular enlarging procedures, but they were not strictly prohibited. Surgeons could do what they needed to do to treat the patient at the time of the procedure.

Your second question is whether there was only left ventricular mass regression in the PPM surgical group?

Dr Totaro. No. I question if it was higher in the surgical group and, more precisely, if left ventricular mass regression was higher in the patients with severe PPM than in the patients without.

Dr Zorn. We saw mass regression with everything, so with TAVR, SAVR, and all subsets with PPM and without PPM, we saw mass regression.

Part of the issue with this trial is we have so few TAVR patients with severe PPM; we do not have enough numbers. There were only 9.

Dr Totaro. I am just talking about the surgical group.

Dr Zorn. We do not see an influence of PPM on mass regression. We see, as a percentage, it is almost identical in the SAVR groups between those with and without PPM.

Dr Totaro. That is a little bit strange. Do you have any idea why patients with severe PPM experienced such an important left ventricular mass regression compared with patients without severe PPM? We could imagine that a patient with severe PPM should not have an important left ventricular mass regression.

Dr Zorn. My only comment is I have been delving into this issue of PPM after SAVR, and it is a morass-there is so much data out there-I do not have the definitive answer as to why we do not see a difference between these groups.

Dr Vinayak Bapat (London, United Kingdom). I am just going to go a bit further on PPM. In TAVR we do not excise the leaflets and in SAVR we excise the leaflets, and your data show very clearly now that despite excising the leaflets we are tending to undersize the valve. Do you think in the future, especially in small adults, we should be looking at newer designs where you can excise the leaflet and you can actually put a bigger valve but that is like a TAVR valve? Do you think that will cause probably less PPM because we are still using the same valves with not much design change for the past 60 years?

Dr Zorn. Agreed, and some of the data presented earlier today would certainly lend some credence to that; they are some interesting data. In truth, an instance of severe PPM of $6 \%$ is relatively low. We can anticipate with further technologic advances that that may even get lower.

One of the thrusts of our study was to find out exactly how important that is. So we do know that severe PPM matters. Any opportunities we have to decrease that are worth exploring. I am not sure we are going to get back to the point where we are going to have to in high-risk patients take out aortic valve leaflets to put a device in. 
TABLE E1. Clinical demographic characteristics for patients with severe prosthesis-patient mismatch (PPM) versus no severe PPM

\begin{tabular}{|c|c|c|c|c|c|c|}
\hline \multirow[b]{2}{*}{ Characteristic } & \multicolumn{3}{|c|}{ TAVR } & \multicolumn{3}{|c|}{ SAVR } \\
\hline & $\begin{array}{l}\text { Severe PPM } \\
\quad(\mathbf{n}=\mathbf{2 4})\end{array}$ & $\begin{array}{l}\text { No severe PPM } \\
\quad(n=343)\end{array}$ & $P$ value & $\begin{array}{l}\text { Severe PPM } \\
\quad(\mathbf{n}=\mathbf{7 5})\end{array}$ & $\begin{array}{l}\text { No severe PPM } \\
\quad(\mathbf{n}=\mathbf{2 5 9})\end{array}$ & $P$ value \\
\hline Age $(y)^{*}$ & $81.5(8.4)$ & $83.1(7.0)$ & .2756 & $82.1(7.0)$ & $83.7(6.1)$ & .0531 \\
\hline Male sex & $11(45.8)$ & $186(54.2)$ & .4253 & $34(45.3)$ & $140(54.1)$ & .1831 \\
\hline Society of Thoracic Surgeons score $(\%)^{*}$ & $7.3(2.9)$ & $7.2(2.9)$ & .8368 & $7.6(2.9)$ & $7.5(3.4)$ & .7779 \\
\hline Body mass index $\left(\mathrm{kg} / \mathrm{m}^{2}\right)^{*}$ & $31.9(7.5)$ & $27.8(6.0)$ & .0016 & $30.4(7.9)$ & $28.0(5.8)$ & .0134 \\
\hline $\begin{array}{l}\text { Logistic European System for Cardiac Operative } \\
\text { Risk Evaluation score }(\%)^{*}\end{array}$ & $17.6(14.5)$ & $17.2(12.5)$ & .8946 & $20.5(15.2)$ & $17.8(11.9)$ & .1615 \\
\hline New York Heart Association functional class III or IV & $22(91.7)$ & $292(85.1)$ & .5517 & $67(89.3)$ & $223(86.1)$ & .4660 \\
\hline Hypertension & $24(100.0)$ & $326(95.0)$ & .2641 & $72(96.0)$ & $250(96.5)$ & .7356 \\
\hline Diabetes mellitus & $11(45.8)$ & $121(35.3)$ & .2975 & $43(57.3)$ & $111(42.9)$ & .0268 \\
\hline Insulin controlled & $3(12.5)$ & $39(11.4)$ & .7462 & $8(10.7)$ & $36(13.9)$ & .4660 \\
\hline Prior stroke & $2(8.3)$ & $43(12.5)$ & .7524 & $10(13.3)$ & $39(15.1)$ & .7012 \\
\hline Coronary artery disease & $17(70.8)$ & $262(76.4)$ & .5380 & $58(77.3)$ & $195(75.3)$ & .7161 \\
\hline Prior coronary artery bypass grafting & $6(25.0)$ & $105(30.6)$ & .5628 & $26(34.7)$ & $76(29.3)$ & .3781 \\
\hline Prior percutaneous coronary intervention & $6(25.0)$ & $118(34.4)$ & .3465 & $32(42.7)$ & $97(37.5)$ & .4140 \\
\hline Severe chronic lung disease & $6(25.0)$ & $43(12.5)$ & .0827 & $8(10.7)$ & $22(8.5)$ & .5623 \\
\hline Home oxygen & $7(29.2)$ & $40(11.7)$ & .0134 & $9(12.0)$ & $28(10.8)$ & .7726 \\
\hline Anemia with prior transfusion & $7(30.4)$ & $57(17.1)$ & .1059 & $8(10.8)$ & $46(18.5)$ & .1209 \\
\hline Albumin $<3.3 \mathrm{~g} / \mathrm{dL}$ & $5(21.7)$ & $51(15.0)$ & .3865 & $6(8.6)$ & $42(16.5)$ & .0968 \\
\hline $5-\mathrm{m}$ Walk test $>6 \mathrm{~s}$ & $17(81.0)$ & $242(78.8)$ & $>.9999$ & $55(85.9)$ & $183(79.9)$ & .2752 \\
\hline Assisted living & $5(20.8)$ & $32(9.3)$ & .0704 & $6(8.0)$ & $31(12.0)$ & .3348 \\
\hline $\begin{array}{l}\geq 2 \text { Katz Index of Independence Activities of Daily } \\
\text { Living deficits }\end{array}$ & $2(8.3)$ & $19(5.5)$ & .6383 & $5(6.7)$ & $19(7.3)$ & .8433 \\
\hline Wheelchair bound & $1(4.2)$ & $13(3.8)$ & $>.9999$ & $7(9.3)$ & $16(6.2)$ & .3419 \\
\hline
\end{tabular}

$T A V R$, Transcatheter aortic valve replacement; $S A V R$, surgical aortic valve replacement; $P P M$, prosthesis-patient mismatch. *Values are presented as mean (SD); all other values are number of patients (\%). 
TABLE E2. Baseline echocardiographic findings for patients with subsequent severe prosthesis-patient mismatch (PPM) versus no severe PPM

\begin{tabular}{|c|c|c|c|c|c|c|}
\hline \multirow[b]{2}{*}{ Characteristic } & \multicolumn{3}{|c|}{ TAVR } & \multicolumn{3}{|c|}{ SAVR } \\
\hline & $\begin{array}{l}\text { Severe PPM } \\
\quad(\mathbf{n}=\mathbf{2 4})\end{array}$ & $\begin{array}{l}\text { No severe PPM } \\
\quad(\mathbf{n}=\mathbf{3 4 3})\end{array}$ & $P$ value & $\begin{array}{c}\text { Severe PPM } \\
(\mathbf{n}=\mathbf{7 5})\end{array}$ & $\begin{array}{l}\text { No severe PPM } \\
\quad(\mathbf{n}=\mathbf{2 5 9})\end{array}$ & $P$ value \\
\hline \multicolumn{7}{|l|}{ Aortic valve } \\
\hline Aortic valve peak velocity $(\mathrm{m} / \mathrm{s})$ & $4.60(0.64)$ & $4.35(0.60)$ & .0512 & $4.37(0.56)$ & $4.38(0.58)$ & .9236 \\
\hline Peak gradient $(\mathrm{mm} \mathrm{Hg})$ & $86.20(23.66)$ & $77.16(21.75)$ & .0511 & $77.69(19.73)$ & $78.04(20.74)$ & .8967 \\
\hline Mean gradient $(\mathrm{mm} \mathrm{Hg})$ & $51.13(15.48)$ & $47.30(14.36)$ & .2094 & $47.79(14.98)$ & $47.79(13.66)$ & .9997 \\
\hline $\begin{array}{l}\text { Aortic valve effective orifice area or } \\
\text { valve area }\left(\mathrm{cm}^{2}\right)\end{array}$ & $0.62(0.12)$ & $0.73(0.23)$ & .0010 & $0.69(0.28)$ & $0.75(0.22)$ & .1664 \\
\hline Effective orifice area index $\left(\mathrm{cm}^{2} / \mathrm{m}^{2}\right)$ & $0.32(0.07)$ & $0.40(0.12)$ & .0005 & $0.36(0.14)$ & $0.41(0.12)$ & .0109 \\
\hline Aortic annulus diameter $(\mathrm{cm})$ & $2.07(0.16)$ & $2.23(0.21)$ & .0006 & $2.14(0.22)$ & $2.19(0.21)$ & .0676 \\
\hline \multicolumn{7}{|l|}{ Left ventricle } \\
\hline End diastolic dimension $(\mathrm{cm})$ & $5.09(0.68)$ & $4.97(0.62)$ & .4292 & $5.12(0.72)$ & $4.98(0.61)$ & .1179 \\
\hline End systolic dimension $(\mathrm{cm})$ & $3.35(0.78)$ & $3.20(0.79)$ & .4668 & $3.42(0.76)$ & $3.23(0.72)$ & .0702 \\
\hline End diastolic volume $(\mathrm{mL})$ & $121.49(45.67)$ & $132.76(50.04)$ & .5093 & $141.15(56.26)$ & $135.06(53.03)$ & .5368 \\
\hline End systolic volume (mL) & $58.37(34.56)$ & $61.90(34.15)$ & .7628 & $73.77(46.78)$ & $60.38(35.23)$ & .1050 \\
\hline Stroke volume $2 \mathrm{DE}(\mathrm{mL})$ calculated & $63.12(19.67)$ & $70.86(27.65)$ & .4088 & $67.38(23.65)$ & $74.67(28.24)$ & .1455 \\
\hline Doppler stroke volume (mL) & $69.60(16.92)$ & $76.50(23.93)$ & .2050 & $69.16(18.06)$ & 77.45 (20.39) & .0025 \\
\hline Left ventricular mass $(\mathrm{g})$ & $226.45(60.41)$ & 225.95 (71.69) & .9781 & $243.18(67.42)$ & $224.68(63.52)$ & .0434 \\
\hline Left ventricular mass index $\left(\mathrm{g} / \mathrm{m}^{2}\right)$ & $119.70(30.64)$ & $122.29(35.50)$ & .7744 & $127.29(33.99)$ & $123.10(33.43)$ & .3790 \\
\hline Ejection fraction $(\%)$ & $56.04(12.25)$ & $58.17(11.25)$ & .3737 & $55.07(13.04)$ & $58.30(11.44)$ & .0380 \\
\hline$\geq$ Moderate mitral regurgitation $(\%)$ & $5(20.8)$ & $31(9.2)$ & .0661 & $10(13.5)$ & $26(10.4)$ & .4541 \\
\hline$\geq$ Moderate total aortic regurgitation $(\%)$ & $1(4.2)$ & $17(5.0)$ & $>.9999$ & $2(2.7)$ & $18(7.1)$ & .2675 \\
\hline Mitral E velocity (m/s) & $1.10(0.35)$ & $1.06(0.32)$ & .5793 & $1.08(0.30)$ & $1.01(0.34)$ & .1576 \\
\hline Mitral E/A ratio & $1.24(0.65)$ & $1.22(0.86)$ & .8890 & $1.28(0.89)$ & $1.07(0.66)$ & .1265 \\
\hline Deceleration time of E (ms) & $231.40(81.50)$ & $226.60(74.07)$ & .7812 & $204.86(64.78)$ & $236.27(80.60)$ & .0020 \\
\hline $\mathrm{E} / \mathrm{e}^{\prime}$ ratio & $28.06(12.46)$ & $24.49(11.87)$ & .2329 & $24.51(10.27)$ & $23.20(10.47)$ & .3989 \\
\hline
\end{tabular}

Categorical variables are presented as n (\%); continuous variables are mean (SD). TAVR, Transcatheter aortic valve replacement; SAVR, surgical aortic valve replacement; $P P M$, prosthesis-patient mismatch; $2 D E$, 2-dimensional echocardiography; $E / A$, earl mitral inflow velocity/late mitral inflow velocity; $E / e^{\prime}$, early mitral inflow velocity/mitral annulus early diastolic velocity.

TABLE E3. Total aortic regurgitation over time according to prosthesis-patient mismatch (PPM) status

\begin{tabular}{|c|c|c|c|c|}
\hline \multirow[b]{2}{*}{$\begin{array}{c}\text { Time and } \\
\text { status }\end{array}$} & \multicolumn{2}{|c|}{ TAVR } & \multicolumn{2}{|c|}{ SAVR } \\
\hline & $\begin{array}{c}\text { Severe } \\
\text { PPM }\end{array}$ & $\begin{array}{c}\text { No severe } \\
\text { PPM }\end{array}$ & $\begin{array}{c}\text { Severe } \\
\text { PPM }\end{array}$ & $\begin{array}{c}\text { No severe } \\
\text { PPM }\end{array}$ \\
\hline \multicolumn{5}{|l|}{$1 \mathrm{mo}$} \\
\hline None & $17.4(4 / 23)$ & $12.1(40 / 330)$ & $74.2(46 / 62)$ & $62.3(149 / 239)$ \\
\hline Trace & $43.5(10 / 23)$ & $41.5(137 / 330)$ & $19.4(12 / 62)$ & $23.8(57 / 239)$ \\
\hline Mild & $30.4(7 / 23)$ & $36.1(119 / 330)$ & $6.5(4 / 62)$ & $12.1(29 / 239)$ \\
\hline Moderate & $8.7(2 / 23)$ & $8.2(27 / 330)$ & $0.0(0 / 62)$ & $1.7(4 / 239)$ \\
\hline Severe & $0.0(0 / 23)$ & $2.1(7 / 330)$ & $0.0(0 / 62)$ & $0.0(0 / 239)$ \\
\hline \multicolumn{5}{|l|}{$1 \mathrm{y}$} \\
\hline None & $36.8(7 / 19)$ & $28.2(78 / 277)$ & $72.3(34 / 47)$ & $66.7(116 / 174)$ \\
\hline Trace & $31.6(6 / 19)$ & $35.7(99 / 277)$ & $21.3(10 / 47)$ & $21.8(38 / 174)$ \\
\hline Mild & $31.6(6 / 19)$ & $28.9(80 / 277)$ & $6.4(3 / 47)$ & $9.8(17 / 174)$ \\
\hline Moderate & $0.0(0 / 19)$ & $6.9(19 / 277)$ & $0.0(0 / 47)$ & $1.1(2 / 174)$ \\
\hline Severe & $0.0(0 / 19)$ & $0.4(1 / 277)$ & $0.0(0 / 47)$ & $0.6(1 / 174)$ \\
\hline
\end{tabular}

Values are presented as $\%(\mathrm{n} / \mathrm{N}) . T A V R$, Transcatheter aortic valve replacement; $S A V R$, surgical aortic valve replacement; $P P M$, prosthesis-patient mismatch. 
TABLE E4. Change in left ventricular (LV) mass and LV mass index

\begin{tabular}{|c|c|c|c|c|}
\hline Characteristic & $\mathbf{P P M}+\mathbf{A R}$ & PPM no AR & No PPM + AR & No PPM no AR \\
\hline SAVR & $\mathrm{n}=0$ & $\mathrm{n}=71$ & $\mathrm{n}=3$ & $\mathrm{n}=232$ \\
\hline \multicolumn{5}{|l|}{ LV mass $(\mathrm{g})$} \\
\hline Baseline & 0 & $243.87(67.18)(\mathrm{n}=61)$ & $228.72(26.28)(n=3)$ & $223.93(61.20)(n=202)$ \\
\hline $1 \mathrm{y}$ & 0 & $215.28(73.28)(\mathrm{n}=36)$ & $178.27(12.48)(\mathrm{n}=2)$ & $188.34(54.17)(\mathrm{n}=132)$ \\
\hline Change & 0 & $-44.04(77.21)(\mathrm{n}=32)$ & $-65.39(18.96)(\mathrm{n}=2)$ & $-41.15(58.15)(\mathrm{n}=119)$ \\
\hline$P$ value for change from baseline to $1 \mathrm{y}$ & NA & .0030 & .1288 & $<.0001$ \\
\hline \multicolumn{5}{|l|}{ LV mass index $\left(\mathrm{g} / \mathrm{m}^{2}\right)$} \\
\hline Baseline & 0 & $128.10(34.55)(\mathrm{n}=61)$ & $125.82(16.10)(\mathrm{n}=3)$ & $122.25(32.13)(\mathrm{n}=202)$ \\
\hline $1 \mathrm{y}$ & 0 & $109.66(38.36)(\mathrm{n}=36)$ & $98.44(4.59)(n=2)$ & $101.42(25.10)(\mathrm{n}=132)$ \\
\hline Change & 0 & $-22.72(39.08)(\mathrm{n}=32)$ & $-36.26(11.33)(\mathrm{n}=2)$ & $-23.44(32.33)(\mathrm{n}=119)$ \\
\hline$P$ value for change from baseline to $1 \mathrm{y}$ & NA & .0025 & .1384 & $<.0001$ \\
\hline TAVR & $\mathrm{n}=3$ & $\mathrm{n}=13$ & $\mathrm{n}=29$ & $\mathrm{n}=193$ \\
\hline \multicolumn{5}{|l|}{ LV mass $(\mathrm{g})$} \\
\hline Baseline & 0 & $205.21(48.65)(\mathrm{n}=10)$ & $271.68(87.16)(\mathrm{n}=26)$ & $216.36(66.54)(\mathrm{n}=170)$ \\
\hline $1 \mathrm{y}$ & $205.71(136.17)(\mathrm{n}=2)$ & $216.85(65.74)(\mathrm{n}=8)$ & $228.58(86.84)(\mathrm{n}=19)$ & $198.96(62.49)(\mathrm{n}=135)$ \\
\hline Change & 0 & $-17.54(83.02)(n=6)$ & $-36.91(59.88)(\mathrm{n}=17)$ & $-17.37(59.72)(\mathrm{n}=122)$ \\
\hline$P$ value for change from baseline to $1 \mathrm{y}$ & NA & .6269 & .0218 & .0017 \\
\hline \multicolumn{5}{|l|}{ LV mass index $\left(\mathrm{g} / \mathrm{m}^{2}\right)$} \\
\hline Baseline & 0 & $111.26(25.69)(\mathrm{n}=10)$ & $146.84(47.58)(\mathrm{n}=26)$ & $117.29(32.39)(\mathrm{n}=170)$ \\
\hline $1 \mathrm{y}$ & $106.09(49.54)(\mathrm{n}=2)$ & $105.58(24.82)(\mathrm{n}=8)$ & $123.87(41.97)(\mathrm{n}=19)$ & $108.03(29.34)(n=135)$ \\
\hline Change & 0 & $-11.04(43.09)(\mathrm{n}=6)$ & $-21.69(31.93)(\mathrm{n}=17)$ & $-10.15(32.29)(\mathrm{n}=122)$ \\
\hline$P$ value for change from baseline to $1 \mathrm{y}$ & NA & .5579 & .0128 & .0007 \\
\hline
\end{tabular}

Values are presented as mean (SD). PPM, Prosthesis-patient mismatch; $A R$, aortic regurgitation; SAVR, surgical aortic valve replacement; $L V$, left ventricular; $N A$, not applicable; $T A V R$, transcatheter aortic valve replacement. 